\title{
Die Beziehungen Theodor Kochers zur «Société internationale de chirurgie»
}

Seine Rolle als deren erster Kongress-Präsident*

von Dorothea Liebermann-Meffert, Martin Allgöwer und Thomas Rüedi

\section{ZuSAMMENFASSUNG}

Mit dem Ziel, dem Fortschritt in der Chirurgie durch freundschaftlichen Erfahrungsaustausch zu dienen, wurde im Jahre 1902 in Brüssel über die engen Grenzen nationalen Denkens hinweg die erste internationale Gesellschaft für Chirurgie gegründet. Als 1905 der erste Kongress stattfand, hatte die "Société Internationale de Chirurgie» (SIC) oder "International Society of Surgery» (ISS) bereits 638 Mitglieder; unter ihnen waren die bedeutendsten Chirurgen aus aller Welt.

Theodor Kocher stand diesem Kongress als Präsident vor und war für seine Thematik verantwortlich. Seine Präsidialrede spiegelt die Ideale der Gesellschaft deutlich wieder. Die persönliche und fachliche Autorität Kochers, sein wissenschaftliches Konzept, das er so gerne seinen Kollegen weitervermittelte, und sein internationales Denken prägten die junge Gesellschaft, in deren Vorstand er bis zu seinem Tod 1917 blieb.

\section{Internationale Begegnungen vor der Jahrhundertwende}

Mit der zunehmenden Möglichkeit chirurgischer Eingriffe wurde auch die wachsende Notwendigkeit eines Erfahrungsaustausches zwischen den Chirurgen offensichtlich. Sie mündete in die Gründung von nationalen Standesgesellschaften wie z.B. des Royal College of Surgeons of England 1843, der deutschen Gesellschaft für Chirurgie 1872 und, sehr spät, der Schweizerischen Gesellschaft für Chirurgie im Jahre 1913.

\footnotetext{
* Diese Arbeit wurde unterstützt von der International Foundation for Postgraduate Surgery.
} 
Über die nationalen Grenzen hinaus begannen um diese Zeit Chirurgen Studienreisen durch Europa zu unternehmen, um an Ort und Stelle die bedeutendsten Chirurgen ihrer Zeit kennenzulernen und operieren zu sehen ${ }^{4}$. So unternahm auch der junge Theodor Kocher (1841-1917) kurz nach Erwerbung seines Arzt- und seines Doktordiploms im Jahre 1865 eine Ausbildungsreise, die ihn nach Berlin, Hamburg, Wien, London und Paris führte. Er hospitierte bei den damals wohl erfahrensten und bekanntesten Chirurgen, so bei Bernhard von Langenbeck, Theodor Billroth, Jonathan Hutchinson, Thomas Spencer Wells, Joseph Lister, Victor Horsley und Auguste Nélaton ${ }^{3,4,13}$.

Der Usus, persönliche Kontakte mit namhaften Kollegen zu knüpfen und Verbindungen zu begründen, erreichte einen Höhepunkt um die Jahrhundertwende. Es kamen nun auch Chirurgen aus den USA nach Europa; zum Beispiel besuchte William Halsted (1852-1922), seinerzeit bereits Professor der Chirurgie am Johns Hopkins Hospital in Baltimore, die berühmten

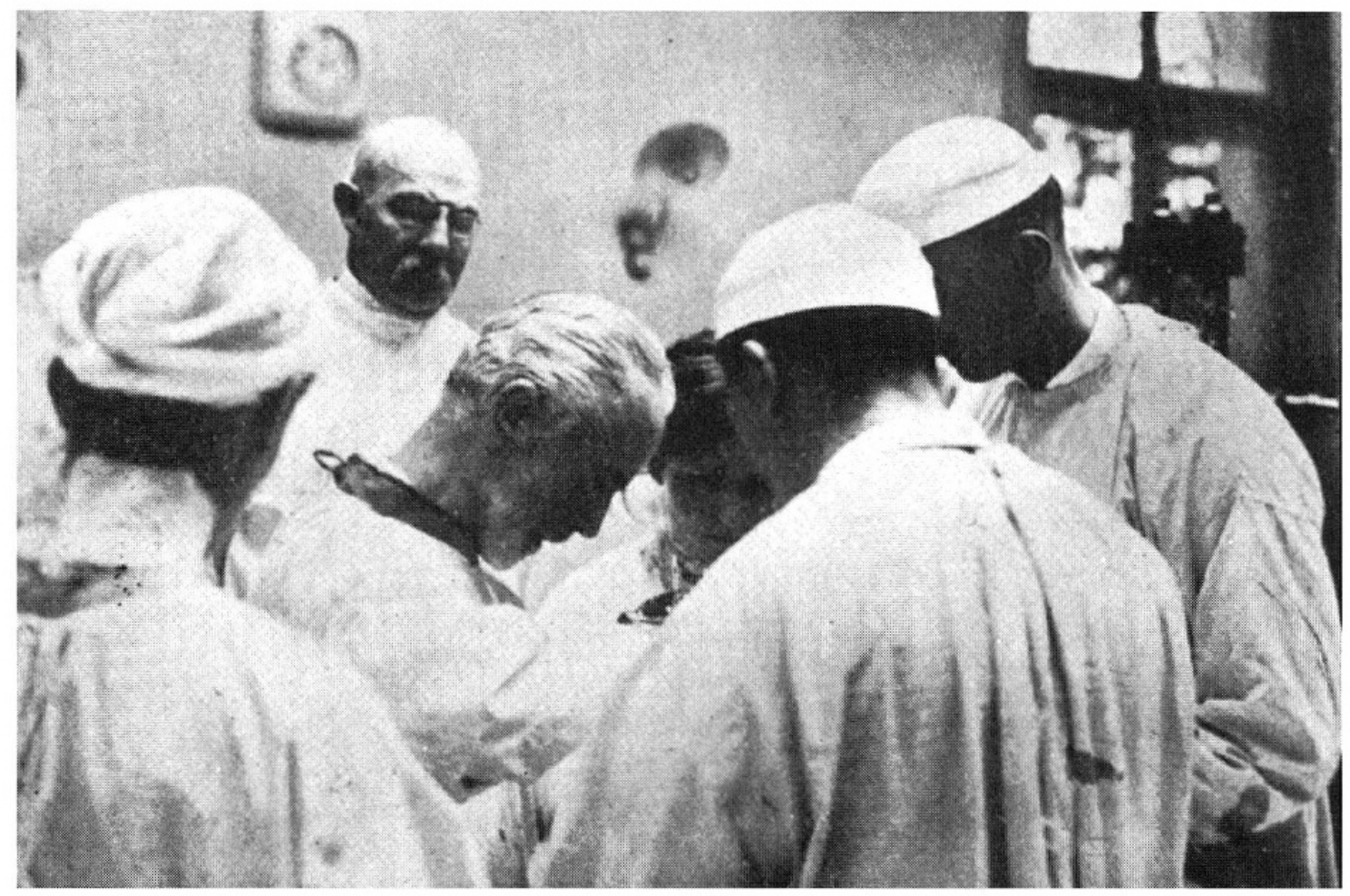

Abb.1: Theodor Kocher bei einer Operation 1913 in Bern mit W. Halsted als Zuschauer (aus Fonio $\left.^{3}\right)$. Beide, Kocher und Halsted, waren seit der Gründung der SIC deren Mitglieder. 
europäischen Kliniken, um Theodor Billroth, Erwin Payr, August Bier, Thomas Spencer Wells und Victor Horsley bei der Arbeit zuzusehen ${ }^{8,10,11,14}$. Halsted besuchte auch Theodor Kocher, den er sehr verehrte, mehrere Male in Bern, um seine Methoden zu studieren (Abb. 1). Beide Chirurgen schienen über Jahre hinweg einen ausgesprochen freundschaftlichen Austausch ihrer Erfahrungen zu pflegen ${ }^{10,11}$. Halsteds Schüler Harvey Cushing (1869-1939) arbeitete mehrere Monate in Kochers Klinik ${ }^{11,14}$.

\section{Gründung einer Gesellschaft mit dem Ziel internationalen Erfahrungs- austausches}

Der so wichtige Austausch chirurgischer Erfahrung über die Grenzen des eigenen Landes hinaus veranlasste eine kleine Gruppe belgischer Chirurgen, die Vorteile zu erwägen, welche die Gründung einer internationalen Gesellschaft für die Chirurgie mit sich bringen würde. Es waren dies die Herren Charles Willems, Professor an der Universität Gent und 1902 Präsident der belgischen Gesellschaft für Chirurgie, Antoine Depage, Professor für Chirurgie an der Universität Brüssel und der Brüsseler Kinderchirurg Professor Jules Lorthioir (Abb.2). Die Verwirklichung des Planes erfolgte bereits $1902^{12}$.

«L'Association fondée à Bruxelles, en 1902, sous le nom de «Société Internationale de Chirurgies a pour but de contribuer aux progrès de la chirurgie, en cherchant à élucider certaines questions d'ordre chirurgical» (Art. 1 der Statuten, Congr. Soc. Int. Chir. 12).

Die Initiative zur internationalen Zusammenarbeit muss sehr rasch aufgegriffen worden sein. Bereits drei Jahre nach der Gründung hatte die «Société Internationale de Chirurgie» oder «International Society of Surgery» (SIC) ISS), wie sie englisch bezeichnet wurde, 638 Mitglieder aus 23 Ländern.

Aus dieser frühen Zeit der Gründung der SIC/ISS fanden wir keine Korrespondenz oder Unterlagen, sondern sind auf Hinweise in den Comptes rendus des 1. Kongressbandes ${ }^{12}$ angewiesen; dass jedoch wirklich eine Notwendigkeit bestand, durch Tagungen das Zusammenkommen zu vereinfachen, zeigt sich darin, dass zahlreiche namhafte Chirurgen aus aller Welt sich der Gesellschaft rasch als Mitglieder anschlossen.

So zeichneten 1905, um ein Beispiel zu nennen, als Mitglieder aus Frankreich Pierre Bazy (Paris), Henri Hartmann (Paris), Mathieu Jaboulay (Lyon), Just Lucas-Champonnière (Paris), Gabriel Tuffier (Paris), Auguste Broca (Paris). 


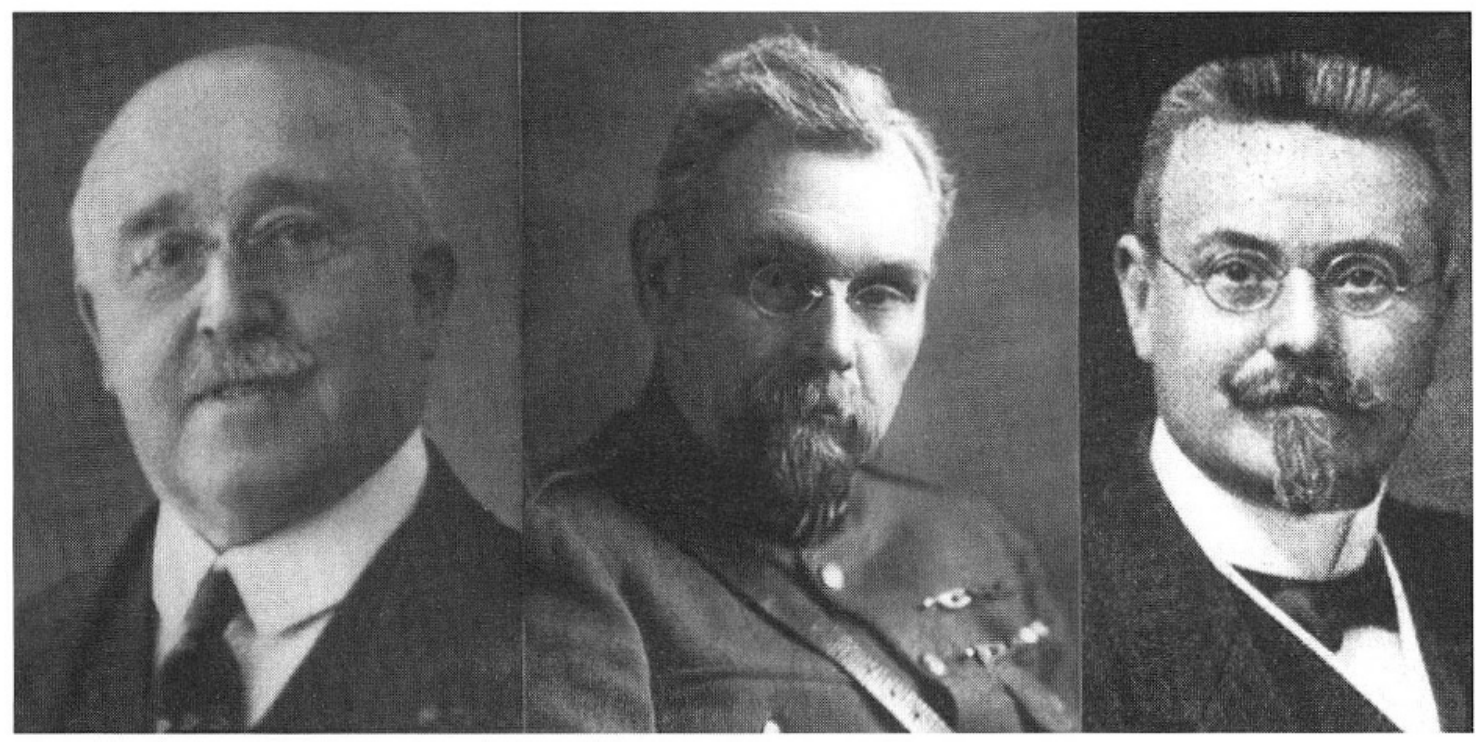

Abb.2: Initiatoren und Gründungsmitglieder der Sociêté Internationale de Chirurgie waren die belgischen Chirurgen Charles Willems aus Gent (1865-1929), Antoine Depage aus Brüssel (18621925) und Jules Lorthioir aus Brüssel (1864-1931), hier von links nach rechts.

Aus den USA waren dabei : George Crile (Cleveland), Harvey Cushing (Baltimore), William Keen (Philadelphia), Rudolf Matas (New Orleans), William Mayo (Rochester), McBurney (New York), John Murphy (Chicago), Roswell Park (Buffalo), Nicholas Senn (Chicago).

Aus Österreich waren es Erwin Payr (Graz), Franz Schloffer (Innsbruck), Anton v. Eiselsberg (Wien).

Aus Italien sind Davide Giordano (Venedig) und Edoardo Bassini (Padua) auf der Liste, aus Deutschland unter anderen Ernst von Bergmann (Berlin), August Bier (Bonn), Heinrich Braun (Göttingen), Vincenz Czerny (Heidelberg), Johann von Esmarch (Kiel), Karl Garrè (Breslau), Paul Kraske (Freiburg), Hermann Kümmel (Hamburg), Hermann Küttner (Marburg), Erich Lexer (Berlin), Werner Körte (Berlin), Ludwig Rehn (Frankfurt), Ferdinand Sauberbruch (Breslau), Friedrich Trendelenburg (Leipzig), Oskar Witzel (Bonn).

Aus Grossbritannien kamen Berkeley Moynihan (Leeds) und Hogarth Pringle (Glasgow), und aus Holland Jan Schoemaker (Den Haag).

Zu den Mitgliedern aus der Schweiz gehörten ausser Theodor Kocher zum Beispiel Carl Arnd (Bern), Heinrich Bircher (Aarau), Ludwig Courvoisier (Basel), Fritz de Quervain (Bern), Ludwig Gelpke (Liestal), Auguste Reverdin (Genève), Jaques Reverdin (Genève), César Roux (Lausanne) und Henri Vuillet (Lausanne).

\section{Kocher als Präsident des ersten internationalen Kongresses für Chirurgie}

Rudolph Matas (1860-1957), Ordinarius für Chirurgie an der Tulane University in New Orleans, welcher seit 1902 Mitglied der Gesellschaft war und 
Kocher persönlich gut kannte, berichtet 1949 in einem historischen Abriss über die SIC/ISS ${ }^{8}$ :

"The first congress was held in Brussels (1905) under the illustrious presidency of Professor Theodore Kocher (1841-1917), of Berne-justly regarded as one of the safest and most skilful surgeons in the world. His clinic at Berne became the surgical Mecca to which patients flocked from all parts of the globe; but, particularly, for the surgical cure of goiter. He performed over a thousand thyroidectomies with the lowest known mortality. His text book on operative surgery, translated into all languages, was the current guide of most surgeons of his day. He was awarded the Nobel prize in 1909, for his research on goiter. Dr. Halsted of Johns Hopkins was his close friend and admirer and spent many of his vacations at his clinic. Harvey Cushing worked in his laboratory and clinic in preparation for his future career as the leading neurosurgeon of his time".

Dies weist auf die Bedeutung der Präsenz des renommierten Theodor Kocher für die Internationale Gesellschaft für Chirurgie hin. Matas' Verehrung für Kocher kommt, nachdem Kocher bereits fast ein halbes Jahrhundert tot war, in diesem Text deutlich zum Ausdruck.

An dem ersten Kongress der SIC/ISS nahmen 195 der 638 Mitglieder teil, das sind $31 \%$. In bezug auf die hohe Zahl der aus der Schweiz kommenden Chirurgen (es handelte sich um ein Drittel der Mitglieder der späteren nationalen Schweizerischen Chirurgischen Gesellschaft, deren Gründungsvorsitz Kocher 1913 inne hatte) ist es durchaus möglich, dass Kocher, welcher die Chirurgie immer in einem internationalen Sinn lehrte, die hohe Anwesenheitszahl beeinflusste.

Bei Gelegenheit des Kongresses fand die Generalversammlung unter Vorsitz Theodor Kochers statt «zur Genehmigung der Abrechnung, zur Neuwahl des Comités, zur Feststellung der auf die Tagesordnung des nächsten Kongresses zu bringenden Themata sowie zur Ernennung der Referenten, endlich zur Wahl des Ortes für den nächsten Kongress und dessen Vorsitzenden» (Art. 9 der Statuten ${ }^{12}$ ). Wir dürfen aufgrund des Wortlautes dieses Artikels annehmen, dass Kocher massgeblich an der Gestaltung des 1. Kongresses und der Wahl der Themen beteiligt war. Die Themata für den Kongress von 1905 lauteten:

1. Valeur de l'examen du sang en chirurgie;

2. Traitement de l'hypertrophie prostatique;

3. Interventions chirurgicales dans les affections non cancéreuses de l'estomac (hiezu hält Kocher einen Diskussionsvortrag ${ }^{7}$ gleichen Titels);

4. Traitement de la tuberculose articulaire (auch hier hält Kocher einen Diskussionsvortrag gleichen Titels);

5. Traitement de la péritonite;

6. Diagnostic des maladies chirurgicales du rein. 
Bei seiner Präsidialrede zur Eröffnung des 1. Kongresses weist Kocher auf Gründungsfakten und Gründe zur Bildung der SIC/ISS hin und führt unter anderem aus ${ }^{5,12 \text { Congressbericht }}$ :

«Nous sommes vraiment heureux de nous réunir dans cette hospitalière Belgique, qui, sous la direction éclairée de son Roi, a souvent montré aux autres nations le chemin du progrès. $[\ldots]$

Je veux évoquer ici, d'abord, le souvenir de Joh. von Mikulicz; nous avions compté sur lui et nous savons qu'il se serait dévoué sans réserve à l'œuvre que nous commençons aujourd'hui. Nous aurions écouté, avec respect, les enseignements que nous promettaient sa longue expérience et cette vaste érudition qui n'avait d'égale que son habileté opératoire. [...] Arthur Gallet a été le promoteur principal de cette réunion, et nous nous souvenons des démarches qu'il a faites, il y a plusieurs années, pour fonder une Société internationale de chirurgie. Vous estimerez avec moi qu'il est juste de rendre hommage à ces collaborateurs enlevés trop tôt à la science et à l'humanité.

Messieurs, l'honneur qu'a bien voulu me faire le Comité international de chirurgie de m'appeler à la présidence du $1^{\text {er }}$ Congrès international de chirurgie me permet de souhaiter la bienvenue à une élite des chirurgiens de tous les pays civilisés et d'être l'interprète des

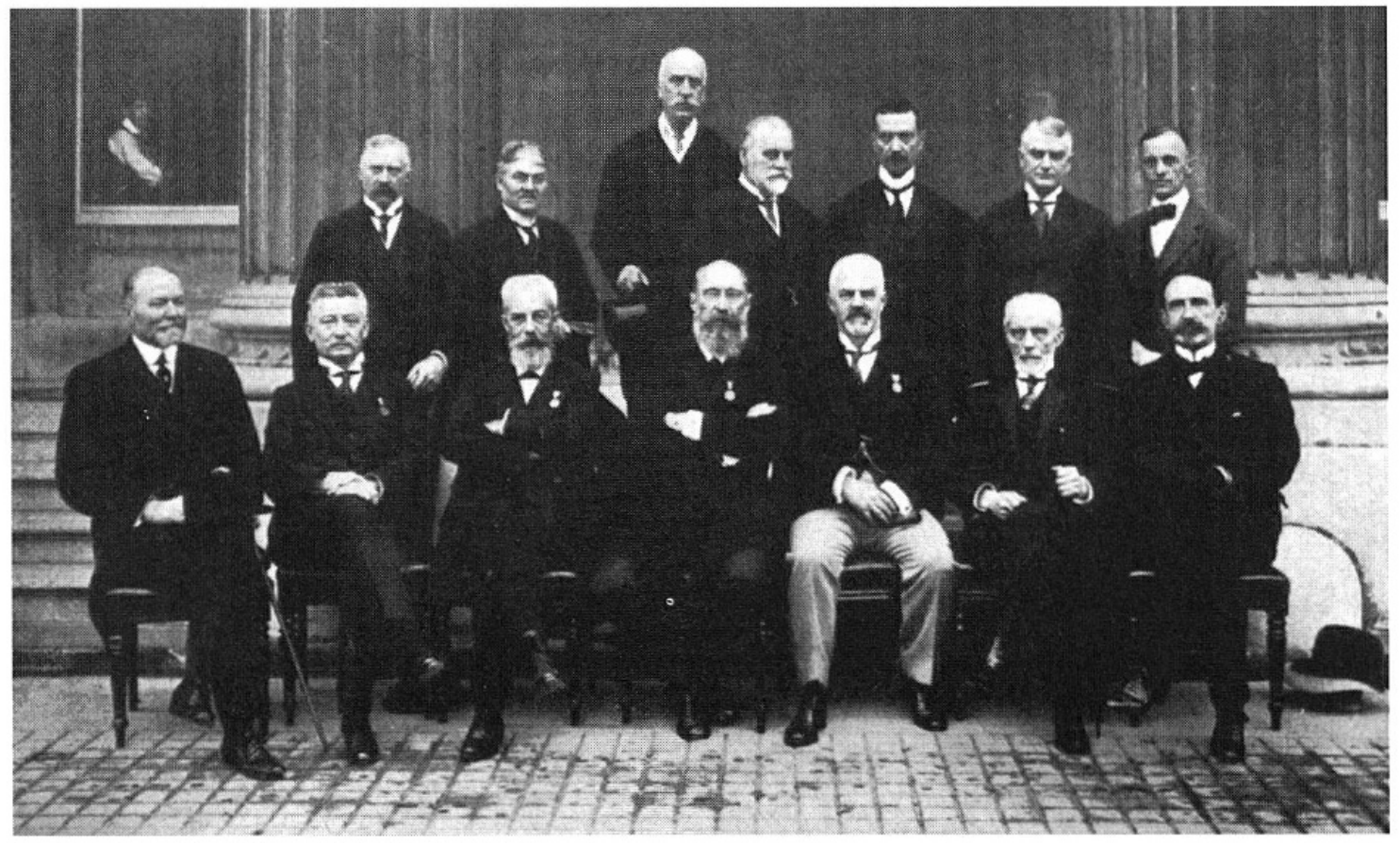

Abb.3: Die neuernannten Ehrenmitglieder des Royal College of Surgeons of England 1913: Sitzend: Murphy*, Körte*, Hartmann*, Fuchs, v. Eiselsberg*, Kocher*, Tuffier*. Stehend: Nicolaysen, Crile*, Bird, Shepherd*,Bastianelli*, W.Mayo*, Cushing*. Von diesen Herren waren die mit * bezeichneten Mitglieder der Internationalen Gesellschaft für Chirurgie. 
vœux les plus sincères pour que cette réunion puisse, dès son entrée au monde, faire preuve de sa capacité aussi bien que de sa ferme volonté de contribuer, pour sa part, au progrès de la vraie science et au bien-être de l'humanité.

Le Comité vous propose de discuter une série de questions qui n'ont rien d'absolument nouveau, qui toutes ont déjà été l'objet de discussions approfondies dans le sein de sociétés nationales. [...] Toutefois, cette perspective d'assister à un tournoi de champions élus ne serait pas suffisante pour justifier l'ambition de ceux qui ont cru devoir convoquer notre réunion. Il faut viser plus haut et nous demander à quels progrès nous aurons à aspirer, qui ne pourraient pas, de la même façon, être accomplis par une assemblée nationale.

La chirurgie a été influencée tout particulièrement pendant ces dernières dizaines d'années par les découvertes des sciences naturelles.

Depuis que nous avons assisté à ce spectacle grandiose d'un bouleversement complet de nos idées sur le traitement des plaies, parce qu'un génie (Lister) a su tirer profit pour la chirurgie des études d'un autre génie (Pasteur) sur les maladies des vins français et des méthodes de prophylaxie contre la décomposition de liquides organiques; depuis que des botanistes consommés (comme Cohn et Koch [sic]), assistés par le perfectionnement de nos microscopes (Abbé), nous ont appris à reconnaître et à classer les (infiniment petits»; depuis la découverte d'agents chimiques et physiques puissants dans une quantité infiniment petite, censée négligeable jusque-là, comme le radium des Curie, des sérums antitétanique, antituberculeux, antidiphtérique des Pasteur, Koch et Behring, les médecins savent guérir des affections qui autrefois faisaient leur désespoir.

Et combien un Röntgen a-t-il dépassé par son ingénieuse découverte tous nos moyens les plus subtils de diagnostic pour certaines lésions chirurgicales!

Grâce à ces découvertes profitant tout particulièrement à la chirurgie, nous avons appris à guérir nombre de maladies inguérissables par la médecine interne. Dans tous les cas où des influences mécaniques jouent un rôle prépondérant dans le développement et le maintien de symptômes pathologiques, c'est le chirurgien qui est appelé à y remédier et à rappeler dans la plupart des cas la santé complète. [...]

Je crois done que la chirurgie opératoire vient d'arriver au point de pouvoir assurer l'heureuse issue d'une opération, vu la sûreté d'arrêter la perte de sang par pinces et ligatures et d'éviter toute infection par une asepsie perfectionnée. [...]»

\section{Es folgt eine mehrere Seiten ausfüllende, ausführliche Stellungnahme und Zusammenfassung der auf dem Kongress vorgetragenen Themen. Dann fährt Kocher fort:}

«Où serions-nous mieux placés qu'ici où un gouvernement bien avisé a convoqué un congrès mondial, qui promet un succès brillant, pour discuter les intérêts les plus divers de la société moderne, en inaugurant une campagne pour l'éradication opératoire du cancer, qui détruit impitoyablement tant d'existences précieuses pour leur famille et leur pays, par les décisions suivantes:

1. Faire préparer par notre Comité ou un comité spécial et, le cas échéant, discuter dans notre assemblée générale une brochure exposant les moyens d'un diagnostic précoce des différentes formes de cancer et la nécessité d'une opération dans les commencements de la maladie; 
2. Envoyer cette brochure à tous les médecins praticiens de tous les pays par l'intermédiaire de comités nationaux au nom de notre Société;

3. Envoyer un extrait de cette brochure à tous les comités de bienfaisance, aussi bien à ces institutions excellentes qui s'appellent chez nous «Gemeinnützige Männer- und Frauenvereines qu'aux associations créées par notre vénéré confrère le Prof. von Esmarch et, s'il est jugé utile, aux instituteurs, pasteurs et curés. [...]

En travaillant énergiquement à l'accomplissement de ce progrès, l'Association internationale de chirurgie ne fait rien que son devoir, en se mettant d'accord avec les deux conditions d'un progrès réel de l'humanité posées par Kant:

«Festhalten an freier Forschung als Bürgschaft strengster Wissenschaftlichkeit, aber auch an einer starken Moral als Grundlage unserer Verantwortlichkeit gegenüber der leidenden Menschheit.»

\section{Kochers Bedeutung für die SIC/ISS}

Nach diesem Kongress hat Kocher nicht mehr an weiteren Tagungen der SIC teilgenommen, blieb jedoch im Vorstand des Internationalen Komitees. Auf der anderen Seite ist anzunehmen, dass Kocher weiterhin rege am Geschehen innerhalb der Gesellschaft interessiert war. So findet sich ein Brief Kochers, datiert «Bern, 28.4. 1914» ${ }^{14}$, bezüglich eines Vortrages beim 4. SIC-Kongress in New York ${ }^{1}$ an Alexis Carrel (1873-1944). Carrel, der für seine Pionierarbeit an Gefässnähten und die Gefäss- und Organtransplantation 1912 den Nobelpreis erhalten hatte, wurde von Kocher um Zusendung des Manuskriptes gebeten:

\footnotetext{
"Dear Sir,

Excuse my troubling you. As I had not the privilege to be able to come to the international Surgical Congress at New York, would you be kind enough to have sent to my address the reports of Carrel, Ceci, Morestin, Payr, Ranzi, Vilard * and the résumé of all reports in four languages.

Especially your report on transplantation would be of the greatest value to me as it is on your success in transplanting tissues that I am basing my hopefulness that we may be able to transplant tissue of thyroid gland from one individual to another-by what Germans call homoio-transplantation.

I have without any doubt seen results by this form of transplantation from one individual to another in man, but the majority of surgeons are absolutely unwilling to accept this fact, notwithstanding the sure results of Cristiani, Kummer and others in Geneva.

Hoping not to trouble you too much by my demand

I am most respectfully yours

T. Kocher"

* which have not been sent to the members who could not go over.
} 
Die Antwort Carrels an Kocher, die vermutlich diesen Brief betrifft, fanden wir bei Morris ${ }^{9}$ :

"Carrel summarized his experience with organ transplantation in a letter to Theodor
Kocher dated May 9, 1914 :
... concerning homoplastic transplantation (from one animal to another) of organs such as
the kidneys, I have never found positive results to continue after a few months, whereas in
autoplastic transplantation the result was always positive. The biological side of the
question has to be investigated very much more and we must find out by what means to
prevent the reaction of the organism to a new organ."

Man mag sich nach diesen Ausführungen fragen, welches sind nun die fassbaren Verdienste Kochers um die SIC/ISS?

Hierzu geben unsere Unterlagen keine Auskunft. Im umfangreichen Schrifttum, welches durch das Sekretariat der SIC/ISS sehr sorgfältig gesammelt wurde und das sich seit 1981 nach dem Transfer des Sitzes der Gesellschaft von Brüssel nach Reinach/Basel dort im Generalsekretariat befindet, fehlen leider alle Originaldokumente bis zum Ende des 1. Weltkrieges. Damit konnte nur sehr wenig über Kochers Wirken für die Gesellschaft verwertet werden, wenn man, wie gesagt, von den aufgeführten Angaben im Kongressbericht absieht. Eine zuverlässige Aussage über den persönlichen Einfluss Kochers auf die Société Internationale de Chirurgie ist daher derzeit nicht möglich.

Dass Kocher aber Kontakte und Austausch mit angesehenen Mitgliedern der Société gehabt haben muss und vermutlich auf diese einen gewissen Einfluss ausübte, ist anzunehmen bei der Betrachtung des Fotos (Abb. 3) der Ehrenmitglieder des Royal College of Surgeons of England von 1913. Auf diesem gehören mit Crile, Sheperd, Bastianelli, Mayo, Cushing, Murphey, Körte, Hartmann, v. Eiselsberg, Kocher, Tuffier immerhin 11 von 14 der dargestellten Ehrenmitglieder der Société an.

Aus den vorhandenen Unterlagen mögen wir rückschliessen, dass die persönliche Autorität Kochers, seine internationale Bedeutung, oder bereits allein die Tatsache, dass er seinen Namen für die Gesellschaft gab und als Vorsitzender des 1.Kongresses hinter ihr stand, für den Start der jungen Gesellschaft sehr wesentlich gewesen ist.

Es ist herauszustreichen, dass in einer Zeit wachsenden politischen Nationaldenkens für das Experiment, eine nicht national gebundene Gesellschaft zu gründen, die Person und das international ausgerichtete Ideengut Kochers eine grosse Unterstützung bedeutete und dass sein wissenschaftliches Konzept die SIC/ISS mit prägte. 


\section{Anmerkungen}

1 Carrel, A., The transplantation of Organs, Congr. Soc. Int. Chir. (CR) 4 (1914), 469-74.

2 von Eiselsberg, A., Lebensweg eines Chirurgen, Innsbruck (Deutscher Alpenverlag) 1940.

3 Fonio, A., Theodor Kocher: Le risque opératoire, Congr.Soc. Int. Chir. (CR) 16 (1955), 276-84.

4 Killian, H., Meister der Chirurgie und die Chirurgenschulen im gesamten deutschen Sprachraum, 2. Aufl., Stuttgart (Thieme) 1980.

5 Kocher, Th., Séance d'inauguration, Congr. Soc. Int. Chir. (CR) 1 (1905), 55-66.

6 Kocher, Th., Traitement de la tuberculose articulaire. Discussion, Quatrième Question, loc. cit., p. 156.

7 Kocher, Th., Traitement des affections non cancéreuses de l'estomac. Discussion, Troisième Question, loc.cit., p. 343.

8 Matas, R., An introduction to the International Society of Surgery : Société Internationale de Chirurgie. Proceed. XIII Congr. SIC, New Orleans 1949, SIC-Archiv, Reinach/Basel

9 Morris, J.B., Schirmer, W. J., The "Right Stuff": Five Nobel prize-winning surgeons, Surgery 108 (1990), 71-80.

10 Rutkow, I. M., William Halsted and Theodor Kocher, an exquisite friendship, Ann. Surg. 188 (1978), 630-7.

11 Rutkow, I. M., Theodor Kocher and his relationship with the American Surgeons Harvey Cushing and William Halsted. In: Boschung, U. (ed.), Theodor Kocher, 1841-1917, Bern (Huber) 1991, pp.41-51.

12 Congrès de la Société Internationale de Chirurgie, comptes rendus 1, 1908, pp. 7-30.

13 Talbott, J. H., A biographical history of medicine. Excerpts and essays on the men and their work, New York (Grune and Stratton) 1979.

14 Tröhler, U., Der Nobelpreisträger Theodor Kocher, 1814-1917. Basel (Birkhäuser) 1984. 


\section{Summary}

\section{Theodor Kocher and the International Society of Surgery-his role as the chairman of its first congress}

With the aim of promoting progress in surgery through the friendly exchange of views and experience, the first International Society of Surgery was founded at Brussels in 1902, hereby helping to overcome the narrow boundaries of that times' nationalism. At its first congress, the "International Society of Surgery (ISS)", otherwise known by its French name "Société Internationale de Chirurgie (SIC)", numbered already 638 members, amongst them the most important surgeons from all over the world.

Theodor Kocher was the president of the first congress, held at Brussels in 1905, and was also responsible for the choice of topics. His presidential address clearly reflected the high aims the Society set itself. Kocher's personal and professional authority, his surgical skill, which he liked so much to communicate to his colleagues, and his internationally minded thinking shaped the young society. He remained in the international committee of the ISS until his death in 1917.

\section{Résumé}

\section{Theodor Kocher et la Société Internationale de Chirurgie - son rôle de président au premier congrès}

La première Société Internationale de Chirurgie (SIC) fut fondée en 1902 à Bruxelles pour faciliter le progrès de la chirurgie par des échanges amicaux à travers les frontières qui séparaient les nations. Kocher fut le président du premier congrès de la $\mathrm{SIC}$, qui se réunit à Bruxelles en 1905. Les auteurs analysent son discours d'ouverture, qui évoque les idéaux et précise les tâches de la Société.

Korrespondenz-Adresse :

Prof. Dr. med. Dorothea Liebermann-Meffert

Departement für Chirurgie, Kantonsspital, Universitätsklinik, Spitalstrasse 21, 4031 Basel

und Chirurgische Klinik und Poliklinik der Technischen Universität München, Klinikum rechts der Isar, Ismaningerstrasse 22, D-8000 München 